\title{
Mon expérience comme agente de quarantaine durant l'accueil des réfugiés syriens
}

\author{
$D^{\prime}$ Amour $\mathrm{R}^{1 *}$
}

\begin{abstract}
Affiliation
${ }^{1}$ Centre des maladies infectieuses d'origine alimentaire, environnementale et zoonotique, Agence de la santé publique du Canada, Ottawa (Ontario)
\end{abstract}

*Correspondance: rolande.damour@phac-aspc.gc.ca

Citation proposée : D'Amour R. Mon expérience comme agente de quarantaine à Montréal. Relevé des maladies transmissibles 2016;42-Suppl 2:S13-4. https://doi.org/10.14745/ccdr.v42is2a04f

Je suis infirmière et j'occupe un poste à l'Agence de la santé publique du Canada depuis presque quinzeans. L'automne dernier, j'ai reçu un courriel demandant des bénévoles pour aller aider les agents de quarantaine durant l'arrivée des réfugiés syriens aux aéroports de Montréal et de Toronto. Je me suis dit : "Pourquoi ne pas sortir de mon cubicule et des sentiers battus, et donner mon nom pour aider? ». J'ai donc soumis ma candidature et suivi peu après la formation d'agent de quarantaine offerte par le Bureau des services de santé à la frontière, de l'Agence. Durant les séances de formation, des agents de quarantaine de profession provenant de différents endroits au pays ont présenté l'élément de base de la formation, à savoir, la Loi sur la mise en quarantaine, que nous devions apprendre et mettre en pratique tout au long de notre formation. Nos journées ont été bien remplies avec des simulations de cas en petits groupes dans lesquels nous discutions de la marche à suivre dans des scénarios variés. Par exemple, nous avons fait l'essai d'un équipement de protection personnelle et procédé à l'ajustement du respirateur N 95. Une fois la formation terminée, je suis retournée à mon poste pour attendre les résultats du processus de sélection.

Quelques semaines se sont écoulées, et comme tout le monde, j'entendais aux bulletins de nouvelles des reportages décrivant l'arrivée des premiers réfugiés syriens au Canada. Je réfléchissais à toute l'organisation que cela exigeait sur tous les plans et je ne pouvais même pas imaginer tout le travail de coordination et de collaboration que cela pouvait comporter.

Le mercredi 30 décembre, j'ai reçu le courriel tant attendu me demandant de me présenter à l'aéroport international Pierre-Elliott-Trudeau de Montréal le mardi suivant. Je partais! J'étais excitée et effrayée en même temps. Serais-je à la hauteur? II fallait donc que je relise mes notes de cours!

Comme un grand nombre de personnes, j'ai voyagé et je suis allée dans plusieurs aéroports pour prendre un vol vers différentes destinations dans le monde. Me préoccupant d'avoir mon billet et mes documents en main, je ne me suis jamais questionnée sur ce qu'il y avait derrière les murs... Eh bien!... il y a toute une équipe de travailleurs qui s'occupe de faire la surveillance afin de prévenir l'entrée de maladies transmissibles au Canada. Cette équipe est composée d'agents de quarantaine qui sont mes collègues à l'Agence. J'en ai fait partie durant quinze jours et voici les grandes lignes de cette expérience.

Avant l'arrivée des vols, nous nous rencontrions dans le bureau des agents de quarantaine pour assister à une séance d'information concernant les particularités du vol. Parfois, les agents de quarantaine avaient un peu d'information telle que le nombre de passagers, la présence de conditions médicales à signaler aux ambulanciers et à mes collègues d'Immigration, Réfugiés et Citoyenneté Canada (IRCC). Ce qui intéressait particulièrement les agents de quarantaine était les passagers chez qui on soupçonnait une maladie transmissible pour pouvoir les rencontrer, les évaluer puis faire un rapport à la santé publique locale pour qu'elle assure un suivi. Après la séance d'information, nous nous sommes rendus à la porte 19, où il y avait un grand espace aménagé pour l'accueil des réfugiés. La première fois que j'y suis entrée, j'ai été impressionnée par le nombre de personnes qui s'y trouvait. Les employés d'IRCC portaient un uniforme jaune fluorescent. Les bénévoles portaient un uniforme rouge et les interprètes, un uniforme bleu. De plus, les douaniers, tous vêtus de noir, se trouvaient dans une section équipée de plus d'une vingtaine de pupitres et d'ordinateurs, d'équipement de biométrie et d'autre matériel de sécurité. II y avait aussi le groupe des premiers répondants composé de pompiers et d'ambulanciers. Les agents de sécurité s'assuraient que seules les personnes autorisées entraient dans la section où I'on travaillait.

Quelques minutes avant l'arrivée du vol, nous attendions avec notre équipe. Les couloirs étaient vides. Nous chuchotions. Nous vérifions nos sacs d'agents de quarantaine pour nous assurer que chaque chose était à sa place, que nos thermomètres tympaniques fonctionnaient, que nous avions suffisamment de questionnaires de rapport de quarantaine et de blocs-notes. Quand près de trois cents passagers de tous âges traverseraient les portes, il faudrait agir rapidement et faire notre travail de façon efficace!

C'est à ce moment-là que je l'ai sentie - cette fébrilité, cette effervescence. Pas seulement moi, mais les interprètes, les bénévoles et mes collègues de IRCC I'ont aussi sentie. Quelque chose de grand allait se produire. Je ne sais pas quoi, mais ça allait être gros. Un douanier est alors venu nous chercher et a demandé à deux agents de quarantaine de monter dans le véhicule de transport de passagers «VTP », ce gros autobus muni d'un mécanisme lui permettant de se hisser dans les airs jusqu'à la porte des gros avions. Tout fin prêts, nous attendions que l'avion atterrisse.

Le VTP s'est approché et nous sommes entrés dans l'avion où le chef des douaniers faisait son travail de sécurité avec l'équipage et où $\mathrm{M}$. Joseph, l'interprète qui n'a manqué aucun vol, faisait une annonce pour souhaiter la bienvenue aux passagers et leur 
demander de demeurer assis pendant que nous ferons notre travail. Nous devions vérifier s'il y avait des gens malades à bord et déterminer avec l'ambulancier et l'équipage s'ils avaient besoin de fauteuils roulants et si nous devions évaluer des passagers malades.

J'ai été surprise lorsque je suis sortie du VTP pour entrer dans l'avion la première fois. II y avait tellement de bruit! Le bruit du moteur du VTP, les annonces au microphone, les discussions entre les douaniers et l'équipage, le sifflement d'air des petites bouches d'aération au plafond, les passagers qui parlaient. II fallait très bien se concentrer et aller à la rencontre des escortes médicales à bord pour qu'ils nous donnent un rapport et nous informent s'il y avait des malades à bord, ce qui signifiait qu'il y aurait du travail pour les ambulanciers et les premiers répondants, et pour nous les agents de quarantaine.

Après avoir discuté avec le personnel médical de l'Organisation internationale des migrations (OIM) qui avait accompagné les réfugiés durant le vol, on annonce que les gens dans les premières rangées pouvaient sortir de l'avion pour monter dans le VTP. Les agents de quarantaine étaient les premiers à observer chaque passager sortant de l'avion pour détecter tout signe de maladie, telle qu'une toux, une éruption cutanée, une fièvre. Le cas échéant, on devait les rencontrer pour prendre leur température et leur poser des questions avec l'aide d'un interprète pour nous assurer qu'ils n'étaient pas atteints d'une maladie transmissible. Les gens arrivaient par le VTP avec leur famille et quelques bagages.

J'avoue que le premier transport de passagers dans le VTP m'a ému car je voyais ces gens qui avaient quitté leur pays et leur vie, pour s'installer au Canada. Nos regards se croisaient. Nos yeux se parlaient. Je leur disais bonjour et bienvenue avec ma voix, et je les rassurais que tout irait bien avec mes yeux. Quant à eux, ils me répondaient de leur regard fatigué mais soulagé. Le sourire est contagieux. C'est un langage universel. Tout le monde était calme - même les enfants.

De la fébrilité avant l'arrivée du vol, nous passions au mode travail, où chacun des douaniers, des interprètes, des bénévoles, des agents de quarantaine, des douaniers, des agents de sécurité, des premiers répondants, faisait son travail spécifique et en collaboration pour suivre les différentes étapes du processus d'accueil.

Au début, le fait de voir des familles nombreuses m'a un peu surpris, cela me rappelait des souvenirs d'enfance, lorsque nos familles étaient plus nombreuses qu'aujourd'hui. Les enfants syriens sont comme tous les enfants : ils sourient quand on leur sourit. Ils répondent d'un signe de la main quand on les salue. Sur chaque vol, il y avait un petit comique, un petit extraverti qui arrivait en nous saluant avec de grands gestes de la main et un sourire immense rempli d'espoir et de reconnaissance. On a tous observé que les aînés des enfants s'occupaient de leurs frères et sœurs quand les parents se levaient de leur chaise pour aller rencontrer les douaniers et ce, pendant de longues périodes parfois. Le processus des douanes est long et tous restaient assis calmement. Il y avait beaucoup d'action, et les familles restaient ensemble et passaient de station en station pour se soumettre aux vérifications.

Mes journées de travail étaient très occupées et je n'avais pas beaucoup de temps pour parler avec mes collègues. J'ai cependant eu l'occasion de parler à quelques bénévoles. Comme moi, ils ont remarqué la collaboration entre les équipes sur place, ils ont été touchés par le côté humain de l'Opération espoir et ils ont beaucoup aimé leur expérience.

Les personnes à qui j'ai le plus parlé sont nos chers interprètes portant l'uniforme bleu qui nous permettaient de bien communiquer avec les voyageurs malades pour leur expliquer que nous allions prendre leur température, leur poser des questions et évaluer les signes et les symptômes qu'ils présentaient, le cas échéant. Chaque détail était important mais il fallait être bref et clair car les passagers avaient fait un long voyage et en avaient encore pour quelques heures avant de partir pour le centre d'accueil où ils allaient recevoir entre autres, des vêtements $d$ 'hiver et leur carte santé et être vus à la clinique, si nécessaire. C'est aussi au centre d'accueil que les réfugiés se joins à leur famille d'accueil ou duquel ils sont partis pour se diriger vers une autre ville au Canada.

J'ai été témoin de petits gestes de gentillesse de la part des douaniers, qui de prime abord, étaient impressionnants tous vêtus de noir et de gilets pare-balles. Bien que les douaniers avaient l'air sérieux et étaient concentrés dans leur travail, ils prenaient tout de même le temps de démontrer de la gentillesse envers les familles. Leur efficacité fut observée et commentée par toutes les équipes sur place.

J'ai remarqué que très souvent, les réfugiés parlaient quelques mots français ou anglais. Quant à moi, je ne connaissais qu'un seul mot arabe "shukran » qui veut dire merci. À un moment, après l'examen d'un enfant enrhumé, j'ai été avisée par une interprète qu'il y avait tout près un enfant montrant des symptômes de fièvre. Ayant terminé mon examen et avec l'aide de l'interprète, j'ai informé la mère que son enfant ne faisait pas de fièvre et n'avait aucun autre symptôme. Nous l'avons encouragée à enlever l'épais manteau et la tuque que son enfant portait, en attendant l'autobus qui les conduirait au centre d'accueil. Avant de partir, elle m'a dit quelque chose que je n'ai pas compris. J'ai répondu « shukran » et elle m'a fait un grand sourire et m'a dit « thank you ».

Ma formation d'infirmière et mon travail à l'Agence m'ont permis de faire partie d'un événement historique. J'ai participé à ma façon au long voyage qu'ont fait les réfugiés syriens pour venir au Canada. Cette expérience est congruente avec ma philosophie de vie personnelle et mes valeurs autant comme infirmière que comme citoyenne canadienne, à savoir, notre réputation d'aider les personnes vulnérables.

Parfois, j'entends un avion qui passe et pendant quelques secondes, je ressens la fierté d'avoir participé à l'Opération espoir. La prochaine fois que je partirai en voyage, je serai la voyageuse au grand sourire à l'aéroport car je sais maintenant ce qui se trouve derrière les murs - des équipes de travailleurs exceptionnels qui sont là pour protéger notre santé et prévenir la propagation de maladies transmissibles. Je remercie grandement mes collègues qui ont été très généreux de leur temps, ce qui m'a donc permis de travailler avec eux. Cela ne doit pas être facile de changer de collègues constamment et de travailler à différentes heures du jour ou de la nuit. Ce fut une expérience que je n'oublierai jamais! Shukran! 